\title{
NBA1, a new player in the Brca1 A complex, is required for DNA damage resistance and checkpoint control
}

\author{
Bin Wang, ${ }^{1,2}$ Kristen Hurov, ${ }^{1}$ Kay Hofmann, ${ }^{3}$ and Stephen J. Elledge ${ }^{1,4}$ \\ ${ }^{1}$ Department of Genetics, Howard Hughes Medical Institute, Center for Genetics and Genomics, Brigham and Women's \\ Hospital, Harvard Medical School, Boston, Massachusetts 02115, USA; ${ }^{2}$ Department of Genetics, The University of Texas M.D. \\ Anderson Cancer Center, Houston, Texas 77030, USA; ${ }^{3}$ Miltenyi Biotec GmbH, 51429 Bergisch-Gladbach, Germany
}

The ability to sense and respond to DNA damage is critical to maintenance of genomic stability and the prevention of cancer. In this study, we employed a genetic screen to identify a gene, $N B A 1$ (new component of the BRCA1 A complex), that is required for resistance to ionizing radiation. The NBA1 protein localizes to sites of DNA damage and is required for G2/M checkpoint control. Proteomic analysis revealed that NBA1 is a component of the BRCA1 A complex, which also contains Brca1/Bard1, Abra1, RAP80, BRCC36, and BRE. NBA1 is required to maintain BRE and Abra1 abundance and for the recruitment of BRCA1 to sites of DNA damage. In depth bioinformatics analysis revealed that the BRCA1 A complex bears striking similarities to the 19S proteasome complex. Furthermore, we show that four members of the BRCA1-A complex possess a polyubiquitin chainbinding capability, thus forming a complex that might facilitate the deubiquitinating activity of the deubiquitination enzyme BRCC36 or the E3 ligase activity of the BRCA1/BARD1 ligase. These findings provide a new perspective from which to view the BRCA1 A complex.

[Keywords: Abraxas; BRCA1 A complex; MPN domain; proteasome; VWA domain]

Supplemental material is available at http://www.genesdev.org.

Received December 8, 2008; revised version accepted February 2, 2009.

Cells have evolved a complex sensory network called the DNA damage response (DDR) to respond to genotoxic stress. The DDR coordinates cell cycle regulation, DNA repair, chromatin reorganization, and transcription in response to damage. Once DNA damage is sensed, a phosphorylation signaling cascade is initiated by the central DNA damage kinases, ATM and ATR (Zhou and Elledge 2000; Harper and Elledge 2007). Recently, a DNA damage-induced ubiquitin signaling pathway has also emerged that works together with phosphorylationdependent signaling to recruit essential repair factors to the sites of DNA damage (Harper and Elledge 2007). Damage-induced ATM/ATR phosphorylation on S139 of histone $\mathrm{H} 2 \mathrm{AX}$ directly recruits MDC1 through MDC1's BRCT domains. MDC1 itself is a substrate of ATM/ATR, and its phosphorylation leads to the recruitment of an ubiquitin ligase RNF8/UBC13 to damage sites. The subsequent ubiqutination events on the damaged chromatin create docking sites for BRCA1 complexes (Huen et al.

${ }^{4}$ Corresponding author.

E-MAIL selledge@genetics.med.harvard.edu; FAX (617) 525-4500.

Article published online ahead of print. Article and publication date are online at http://www.genesdev.org/cgi/doi/10.1101/gad.1770309. Freely available online through the Genes \& Development Open Access option.
2007; Kolas et al. 2007; Mailand et al. 2007; Wang and Elledge 2007).

The BRCA1 tumor suppressor is associated with hereditary breast and ovarian cancer and plays critical roles in DNA repair, cell cycle checkpoint control, and maintenance of genomic stability (Venkitaraman 2002; Narod and Foulkes 2004). BRCA1 contains two C-terminal BRCT repeats that bind pSPxF phospho-amino acid motifs and an N-terminal RING domain that binds BARD1, forming an E3 ubiqutin ligase (Wu et al. 1996; Hashizume et al. 2001; Ruffner et al. 2001; Baer and Ludwig 2002). We and others found that BRCA1 forms at least three distinct complexes-the BRCA1 A, BRCA1 B, and BRCA1 C complexes-through association with different adaptor proteins via their $\mathrm{pSPxF}$ motifs (Yu and Chen 2004; Greenberg et al. 2006; Wang et al. 2007). The adaptor proteins are Abraxas (Abral) for the BRCA1 A complex, Bach1/Brip1 for the BRCA1 B complex, and CTIP for the BRCA1 C complex and each complex forms in a mutually exclusive manner consistent with the fact that they occupy the same physical location on Brcal. The BRCA1 A complex contains BRCA1/BARD1, Abraxas, Rap80, and BRCC36, a deubiquitination enzyme (Dong et al. 2003; Sobhian et al. 2007; Wang and Elledge 2007). In this complex, Abraxas contains a phosphorylated 
SPxF motif at its $C$ terminus that associates with the BRCT domain of BRCA1 (Wang et al. 2007). Abraxas mediates the interaction of both Rap 80 and BRCC36 with BRCA1 (Wang and Elledge 2007). Abraxas associates with Rap80 through the N-terminal domain (Wang and Elledge 2007). Rap80 contains two UIM (ubiquitin-interacting motif) domains that have been shown to bind K63-linked ubiquitin chains (Sobhian et al. 2007; Yan et al. 2007), and are required for the localization of the BRCA1 A complex to ionizing radiation (IR)-induced foci (IRIFs) (Kim et al. 2007; Sobhian et al. 2007; Wang et al. 2007). Abraxas binds BRCC36 through the coiled-coil domains on both proteins (Wang and Elledge 2007). BRCC36 is a deubiquitination enzyme that specifically catalyzes K63-linked polyubiquitin chains and contains a Mpr-1/Pad-1 N-terminal $\left(\mathrm{MPN}^{+}\right) / \mathrm{JAMM}$ domain (Dong et al. 2003; Sobhian et al. 2007).

In this study, using genetic and proteomic approaches we identified a new component of the BRCA1 A complex, NBA1. NBA1 plays a critical role in IR resistance, cell cycle regulation, and the recruitment of BRCA1 to sites of DNA damage. In depth bioinformatics analysis of the components of the A complex reveal that it has striking parallels to the lid complex of the $26 \mathrm{~S}$ proteasome. Furthermore, we show that several members of the BRCA1A complex possess a polyubiquitin chain-binding capability, thus forming a complex that might facilitate the deubiquitinating activity of the deubiquitination enzyme BRCC36 or the E3 ligase activity of the BRCA1/BARD1 ligase.

\section{Results}

Identification of a novel protein from an IR sensitivity screen

We recently developed bar-coded, microRNA-based shRNA libraries targeting the entire human genome that can be expressed from retroviral vectors for stable gene knockdown (Paddison et al. 2004; Silva et al. 2005; Schlabach et al. 2008; Silva et al. 2008). In this study, we performed a small scale loss-of-function screen to uncover novel players in the mammalian DDR pathway utilizing complex pools of shRNAs coupled with microarray deconvolution technology. Our screen is designed to identify genes whose inhibition leads to cellular sensitivity to IR (Fig. 1A). A pool of shRNAs was introduced into U2OS cells via retroviral transduction from an MSCV-driven vector at an approximate multiplicity of infection of 1 (Schlabach et al. 2008; Silva et al. 2008). Following selection of the cells with puromycin, the cells were treated with IR or left untreated. The cells were passaged in triplicate for $\sim 12$ population doublings (14 d) and then harvested for genomic DNA isolation. The halfhairpin sequence was PCR recovered and the DNA from untreated cells and IR-treated cells were labeled with Cy5 and $\mathrm{Cy} 3$, respectively, and competitively hybridized to a custom microarray containing half-hairpin probes. Any shRNA that silences a gene that is necessary for the cell to respond to or repair DNA double-strand breaks will be deleterious and will drop out of the IR-treated population.
In a preliminary screen, we identified two different shRNAs that target a previously uncharacterized ORF, c19orf62 (Fig. 1B), also noted as a hypothetical protein HSPC142. We will refer to c19orf62 as NBA1 for reasons described below.

To validate that NBA1 shRNAs lead to increased IR sensitivity, cells were infected with retroviruses expressing the individual shRNA and viability in response to IR was tested using a multicolored cell competition assay (Smogorzewska et al. 2007). Cells expressing the NBA1 shRNAs and dsRed were mixed equally with uncolored cells expressing a control shRNA that targets firefly luciferase. The mixed cells were treated with IR or left untreated and the viability of the dsRed-expressing cells was monitored by fluorescence-activated cell sorting (FACS) relative to untreated cells (Fig. 1C). Both NBA1 shRNAs, as well as an ATM shRNA, led to decreased cell viability in response to IR. Immunoblots of NBA1 protein levels show the efficiency of shRNA knockdown (Fig. 1C). To further address the specificity of the shRNA knockdown and to rule out that off target effects lead to the IR sensitivity phenotype, we also tested siRNAs that target distinct regions of the NBA1 sequence in an independent assay. Three siRNAs, which efficiently knocked down NBA1 expression, also led to increased IR sensitivity in a clonogenic survival assay in U2OS cells (Fig. 1D). In addition, when we expressed an NBA1 cDNA lacking the shRNA target site in cells treated with NBA1 shRNA\#1, the shRNA-resistant cDNA reversed the IR sensitivity of the NBA1-shRNA (Fig. 1D). Furthermore, we found that depletion of $N B A 1$ rendered cells more sensitive to other DNA-damaging agents, such as camptothecan (CPT), UV, and Mitomycin C (MMC) (Supplemental Fig. S1).

\section{NBA1 localizes to sites of DNA damage}

Proteins with roles in IR resistance can act directly or indirectly in the sensing and repair of DNA damage. Proteins acting in a direct manner in these processes often localize to chromatin at the sites of DNA damage. To investigate the localization of NBA1, we fused NBA1 in frame with a N-terminal GFP tag in MSCV. We found that in virally infected cells, GFP-NBA1 was localized in both the nucleus and cytoplasm, however, when extracted prior to fixation, the nuclear portion of GFPNBA1 relocalized to foci in response to IR (Fig. 2A). To verify that these were indeed sites of DNA damage, we performed two experiments. We first examined colocalization with Brcal, which has been shown previously to localize to damage sites. We found that NBA1 colocalizes with Brcal at sites of IR-induced damage (Fig. 2B). Second, we examined localization using a UV laser to microirradiate cells and found that NBA1 also localized to the laser-induced DNA damage stripes that overlaps with localization of histone $\gamma-\mathrm{H} 2 \mathrm{AX}$ (Fig. 2C). These results indicate that NBA1 is likely to play a direct role in DNA damage sensing and/or repair.

\section{NBA1 is a new component of the BRCA1 A complex}

To explore the function of NBA1, we performed a tandem affinity purification (TAP) on 293T cells stably expressing 

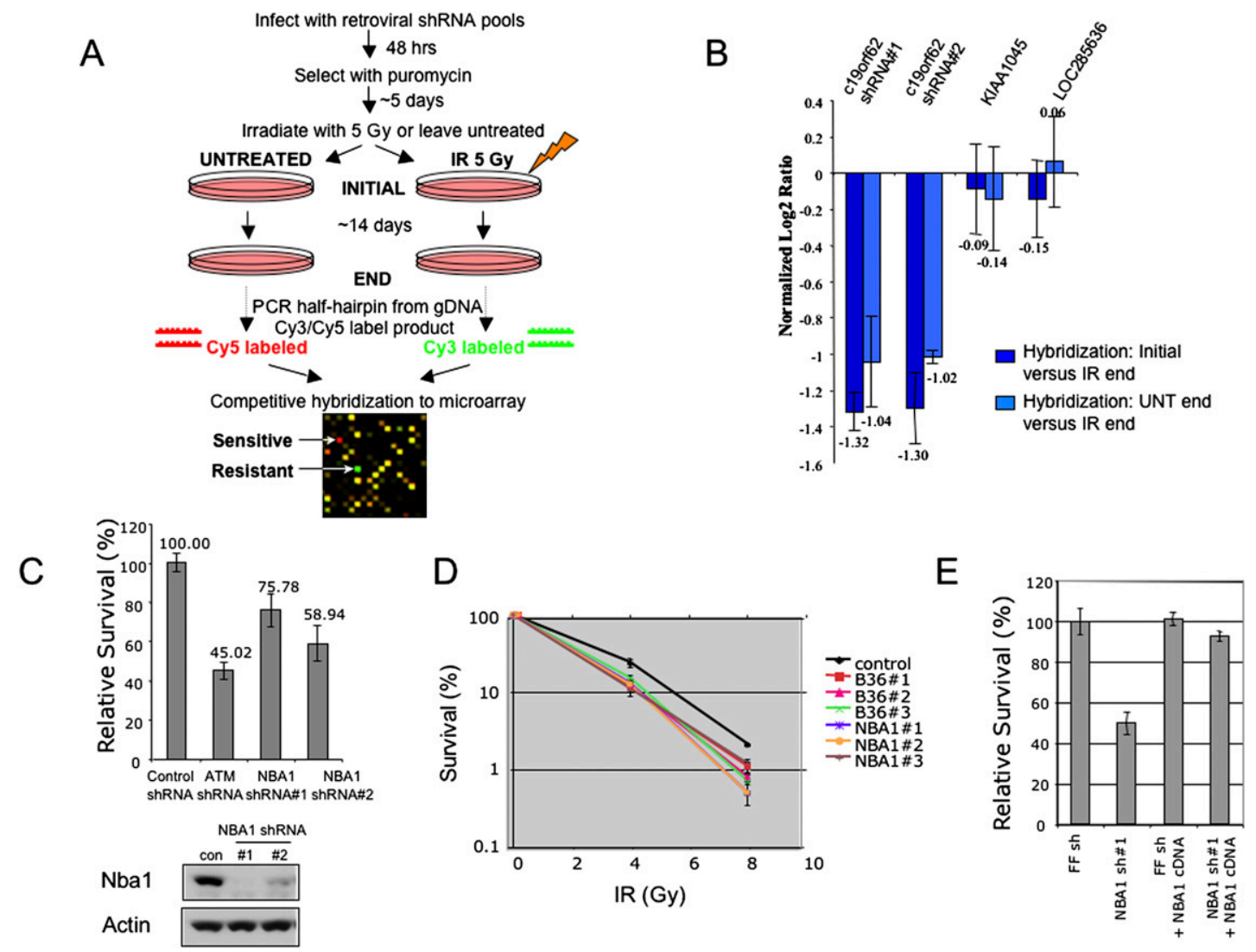

Figure 1. NBA1 is required for radioprotection. $(A)$ Overview of the pool-based shRNA screen for regulators of the DNA double-strand break response in mammalian cells. $(B)$ Identification of NBAl as a gene required for IR resistance. shRNAs are graphed based on their mean normalized $\log 2 \mathrm{Cy} 5 / \mathrm{Cy} 3$ ratio, with a ratio less than -1 indicating a decrease in abundance greater than twofold. Two shRNAs targeting distinct regions of the NBA1 sequence scored in the screen. Two unrelated shRNAs that were considered unchanged are also shown for comparison. Error bars represent standard deviation across triplicates. $(C)$ Validation of NBA1 shRNAs that lead to increased IR sensitivity. shRNAs were individually packaged into retroviruses and infected into cells in independent triplicates. Cells were either treated with 3 Gy IR or left untreated and cell viability was monitored $\sim 9 \mathrm{~d}$ later. An ATM shRNA that also scored in the screen and led to IR sensitivity is shown for comparison. $(D)$ Validation of the NBAl IR sensitivity phenotype using siRNAs. Three different pairs of siRNAs against NBA1 gene and BRCC36 gene were transfected into U2OS cells. Cell survival was measured by colony-forming assay. (E) NBA1 cDNA resistant to shRNA rescues IR sensitivity. U2OS cells expressing a control firefly shRNA or NBA1 shRNA\#1 was infected with virus expressing a shRNA\#1-resistant NBA1 cDNA or a control vector construct and cells were tested for sensitivity to IR.

Flag-HA-tagged NBA1. In the analysis of the associated proteins we identified a number of proteins including Abra1 (Fig. 3A; Supplemental Table S1). Abra1 is known to associate with Brcal. Consistent with an association with Abra1, we identified BRCA1 in the NBA1 pull-down by Western blotting (Fig. 3B). To further confirm this association we performed a purification of Abral-associated proteins using TAP on 293T cells stably expressing endogenous levels of Flag-HA-tagged Abral using mass spectrometry analysis, all of the known components of the BRCA1 A complex were identified including BRCA1/ BARD1, Rap80, BRE(BRCC45), and BRCC36 (Fig. 3C). Importantly, NBA1 was also identified as a prominent and potentially stoichiometric band in the Abral protein complex (Fig. 3C). Given its membership in the BRCA1 A complex we named this ORF NBA1. We then tested whether NBA1 associates with the known players in the A complex of BRCA1. We found that endogenous NBA1 associates with both Abra1 and Rap80 (Fig. 3D).
We also found that NBA1 seems to be required to maintain the abundance of BRE and Abral as reduction of NBA1 results in a decrease in BRE and Abral levels (Fig. 3E). Like GFP-tagged BRE, immunoprecipitation of GFP-tagged NBA1 revealed that it associates with each member of the Brcal A complex suggesting that all of the proteins are present in one complex as opposed to multiple different Abral-containing complexes (Fig. 3F).

\section{NBA1 is required for the DNA damage checkpoint}

To explore the role of NBA1 in the DDR we examined the ability of cells depleted for NBA1 to arrest the cell cycle in G2 in response to IR, a function known to be dependent on Brca1. NBA1-depleted cells were unable to arrest efficiently in G2 in response to IR (Fig. 4A). This is similar to defects in cells depleted for another component of the Brcal A complex, BRCC36 (Fig. 4A). This suggests that NBA1 plays an integral role in the function of the Brcal A complex. 
Wang et al.

A

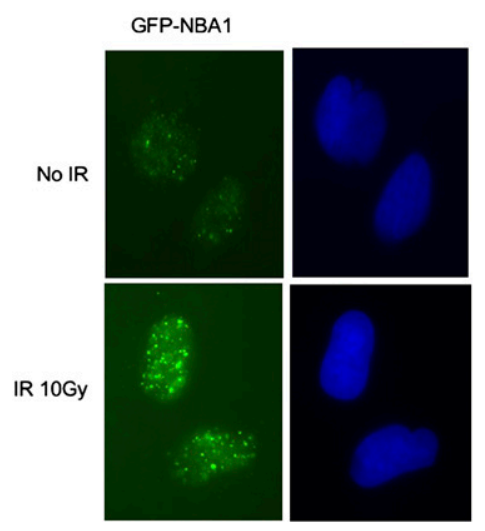

B

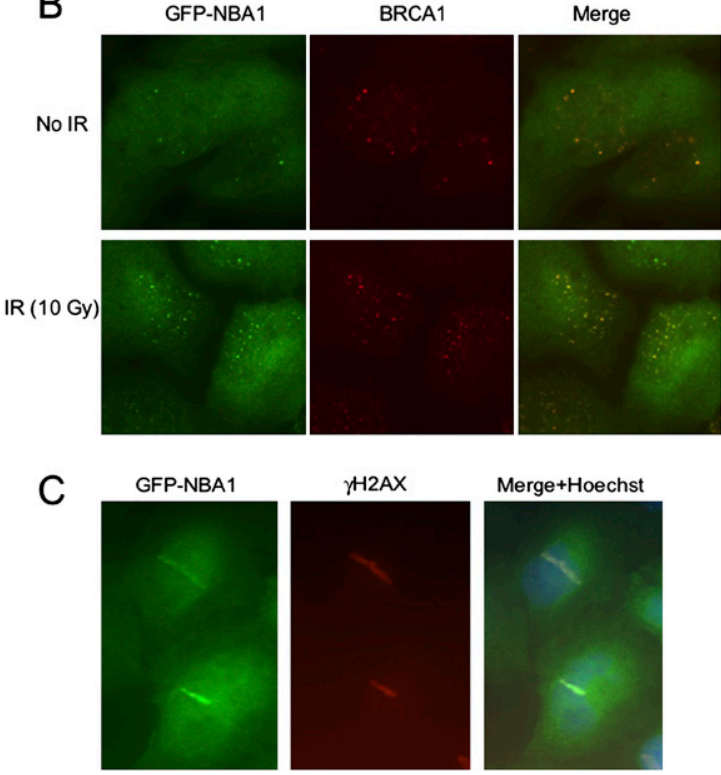

Figure 2. NBA1 accumulates to DNA damage sites in response to IR. (A) NBA1 localizes to IRIF. U2OS cells that stably express a GFPNBA1 fusion were treated with 10 Gy IR followed by a 4-h incubation at $37^{\circ} \mathrm{C}$. Cells were then extracted with $0.5 \%$ Triton X-100, fixed with $3.7 \%$ formaldehyde, and stained with an anti-GFP antibody and Hoechst dye. (B) GFP-NBA1 forms IRIF that colocalize with BRCA1 foci. U2OS cells stably expressing GFP-NBA1 protein were treated with 10 Gy IR. After $2 \mathrm{~h}$ incubation of cells post-IR, cells were fixed and stained with antibodies against GFP and BRCA1. (C) GFP-NBA1 accumulates to laser-induced DNA damage regions that colocalize with $\gamma$-H2AX. U2OS cells stably expressing GFP-NBA1 were treated with a laser for microirradiation.

\section{NBA1 is required for proper localization of BRCA1} protein in response to $I R$

Given NBA1's central role in IR resistance and G2/M checkpoint control, we wished to determine what other functions of the Brcal A complex were dependent on NBA1. Therefore, we examined whether NBA1 is required for Brcal foci formation in response to IR. Similar to BRCC36 and BRE, we found that NBA1 is also required for optimal foci formation of BRCA1 in response to IR. In siRNA-treated cells lacking NBA1 expression, the percentage of cells forming IR-induced BRCA1 foci was decreased (Fig. 4B). This phenotype could be rescued by expression of a NBA1 cDNA that lacks the shRNA targets site present in the $3^{\prime}$ untranslated region (UTR) (Fig. 4C). Like Brca1, when NBA1 was depleted, Rap80 foci formation was also compromised, and this could also be rescued with the shRNA-resistant NBA1 cDNA (Supplemental Fig. S2). Therefore, NBA1 is a critical component of the BRCA1 A complex required for BRCA1/ BARD1 E3 ligase recruitment to sites of DNA damage.

\section{NBA1 is associated with the BRCA1 A complex through the ABR domain of Abra1}

To explore how NBA1 is associated with the Brcal A complex, we began a deletion analysis of the Abra1 protein, which appears to act as a scaffold for the A complex. Abral is known to mediate the interaction of Rap80 with BRCA1 (Wang et al. 2007). In addition BRCC36 also directly interacts with Abral through its coiled-coil domain (Wang and Elledge 2007). We found that, similar to $\mathrm{BRE}, \mathrm{NBA} 1$ is incorporated into the Brcal A complex via the N-terminal region of Abral, through analysis of a series of deletion mutants of Abra1 (Fig. 5A,B; Wang and Elledge 2007). In addition, Abral mediates the interaction of NBA1 and BRE with Rap80 and BRCC36 (Fig. 5C-E; Wang and Elledge 2007). Thus, Abral binds multiple A complex components along its length; its $\mathrm{N}$-terminal ABR domain binds NBA1 and BRE, the central coiled-coil domain binds BRCC36, the phosphorylated p-Ser406 binds Brcal and the large $\mathrm{N}$-terminal region spanning both $\mathrm{ABR}$ domain and the coiled-coil domain and beyond is responsible for binding to Rap80 (Fig. 5F). Therefore, Abral serves as a central organizing adaptor protein that bridges the interaction of each member of the BRCA1 A complex.

\section{Similarities between the $19 S$ proteasome complex proteins and the Brca1 A complex components revealed through bioinformatic analysis}

Bioinformatics analysis of the NBA1 protein revealed the presence of a VWA (von Willebrand factor A) domain in its $\mathrm{N}$ terminus (Fig. 6A) that is homologous to the VWA domain of the proteasome subunit Rpn10/PSMD4/S5a (Hofmann and Bucher 1998; Hofmann and Falquet 2001). The VWA domain of Rpn10/S5a/PSMD4 is required for maintaining the lid-base association of the $19 \mathrm{~S}$ regulatory particle of the proteasome and certain aspects of proteasome function (Glickman et al. 1998; Fu et al. 2001; Verma et al. 2004; Hamazaki et al. 2007).

The identification of the NBA1 VWA domain suggested a connection of the Brcal A complex with the proteasome lid. This prompted us to perform a more thorough bioinformatics analyis of all of the components of the Brcal A 


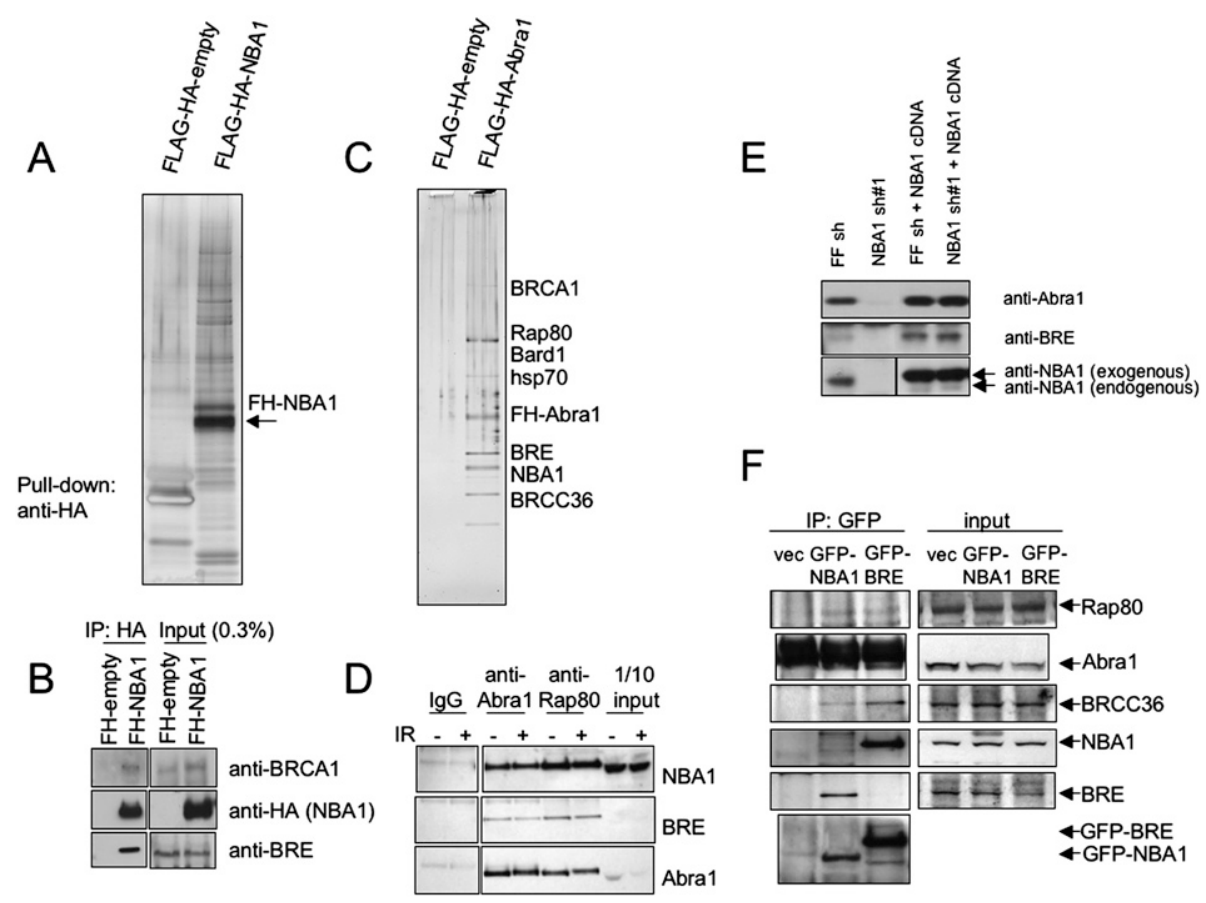

Figure 3. NBA1 is a BRCA1 A complex component. (A) TAP purification of Flag-HA-NBA1. (B) NBA1 interacts with BRCA1. Immunoprecipitated Flag-HA-tagged NBA1 was fractionated in the gel and probed with antibodies against BRCA1. (C) TAP purification of Flag-HA-Abral. $(D)$ Endogenous NBAl interacts with Rap80 and Abra1. Lysates from 293T cells treated with 10 Gy IR follwed with $2 \mathrm{~h}$ incubation or untreated were used for immunoprecipitation with antibodies against Abral or Rap80, franctionated with SDS-PAGE gel and Western blot was carried with antibodies against Abra1, Rap80, BRE, or NBA1. (E) NBA1 controls BRE and Abra1 abundance. U2OS cells were infected with control firefly (FF) hairpin or shRNA\#1 targeting the 3'UTR region of NBA1 or $2 \mathrm{~d}$ later infected again with NBA1 cDNA lacking the 3'UTR region. Cell extracts were immunoblotted for Abral, BRE, and NBA1 protein levels. $(F)$ GFP-tagged NBA1 interacts with each member of the BRCA1-A complex. 293T cells stably expressing GFP-NBA1 or GFP-BRE were used to prepare lysates for the immunoprecipitation with antibodies against GFP. The immunoprecipitates were then separated in SDS-PAGE gel and tested for binding to Rap80, Abra1, and BRCC36 using Western blot and antibodies against Rap80, Abra1, BRCC36, BRE, and NBA1.

complex to search for more motifs that had escaped earlier detection. This analysis revealed that Abral, and its paralog Abro1, contains a MPN domain in its $\mathrm{N}$ terminus. MPN domains are found in a group of proteins with a variety of functions: proteasome regulatory subunits, eukaryotic initiation factor 3 (eIF3) subunits, the signalosome, and regulators of transcription factors (Hofmann and Bucher 1998; Maytal-Kivity et al. 2002; Verma et al. 2002; Yao and Cohen 2002; Cope and Deshaies 2003). BRCC36 contains a $\mathrm{MPN}^{+} / J A M M$ domain at its $\mathrm{N}$ terminus that is simailar to the Rpn11/PSDE of the poteasome lid complex and the Csn5 subunit of the COP9 signalosome, and displays a deubiqutination enzyme activity (Dong et al. 2003; Sobhian et al. 2007). Through protein sequence analysis of different species of Abral, Abrol, and previously identified MPN domains including Rpn8/PSD7 subunit of the proteasome lid complex and Csn6 of the COP9 signalosome, we found that the MPN domain in Abral and Abrol lacked conserved residues that coordinates $\mathrm{Zn}^{2+}$ binding required for enzymatic cleavage activity (Fig. 6B), and thus not likely to possess an enzymatic activity like the $\mathrm{MPN}^{+} / \mathrm{JAMM}$ domain (Ambroggio et al. 2004).

Bioinformatics analysis also revealed that BRE contains two UEV domains lacking the critical cysteine required for ubiquitination (Fig. 6C). Since both MPN and UEV domains are potential ubiquitin-binding domains (Hurley et al. 2006), we then tested whether Abral, BRE, and BRCC36 possess the capacity to bind ubiquitin chains (Fig. 7A). In an in vitro ubiquitin-binding assay, the indicated GST-tagged proteins were purified from bacteria and incubated with either K63linked polyubiquitin chains or K48-linked polyubiquitin chains. We found that purified Gst-tagged Abral, Abrol, BRCC36, or BRE all bind to polyubiquitin chains with at least eight-ubiquitin subunits, both K63-linked and K48linked (Fig. 7A). In comparison, wild-type UIM domains of Rap80 binds specifically only to K63-linked polyubiquitin chains of various lengths as shown previously (Fig. 7A; Sobhian et al. 2007; Yan et al. 2007). Therefore, four members of the BRCA1 A complex, Abral, BRCC36, BRE, and Rap80 all possess polyubiquitin chain-binding activity, with Rap80 providing a binding specificity of K63-linked polyubiquitin chains for the complex.

\section{Discussion}

NBA1 is a critical component of the BRCA1 A complex

In this study we identified a gene, $N B A 1$, that is required for resistance to IR. NBA1 localizes to sites of DNA damage and regulates the IR-induced G2-M checkpoint. Through mass spectrometry analysis we discovered 
Figure 4. (A) NBA1 is required for proper G2-M regulation in response to IR. U2OS cells were untreated or treated with 3 Gy $\mathrm{IR}$, then incubated for $1 \mathrm{~h}$ at $37^{\circ} \mathrm{C}$ before fixation and $\mathrm{p}-\mathrm{H} 3$ antibody staining. Two independent experiments were performed with two different pairs of siRNAs to NBA1 and BRCC36. $(B)$ NBAl is required for optitmal BRCA1 and Rap80 foci formation in response to IR. U2OS cells were transfected with control or siRNAs against NBA1, BRCC36, or BRE for $2 \mathrm{~d}$, then irradiated with 10 Gy IR, incubated for $2 \mathrm{~h}$, fixed and immunostained with the indicated antibodies. (C) The defect in BRCA1 IRIF formation in cells expressing shRNA to NBAl can be rescued by expression of an shRNA-resistant NBA1 cDNA. U2OS cells expressing NBA1 shRNA\#1 were infected with virus expressing NBA1 cDNA lacking the target sequence of shRNA\#1, irradiated with 10 Gy IR, incubated for $4 \mathrm{~h}$, fixed, and stained with Brcal antibodies.

A
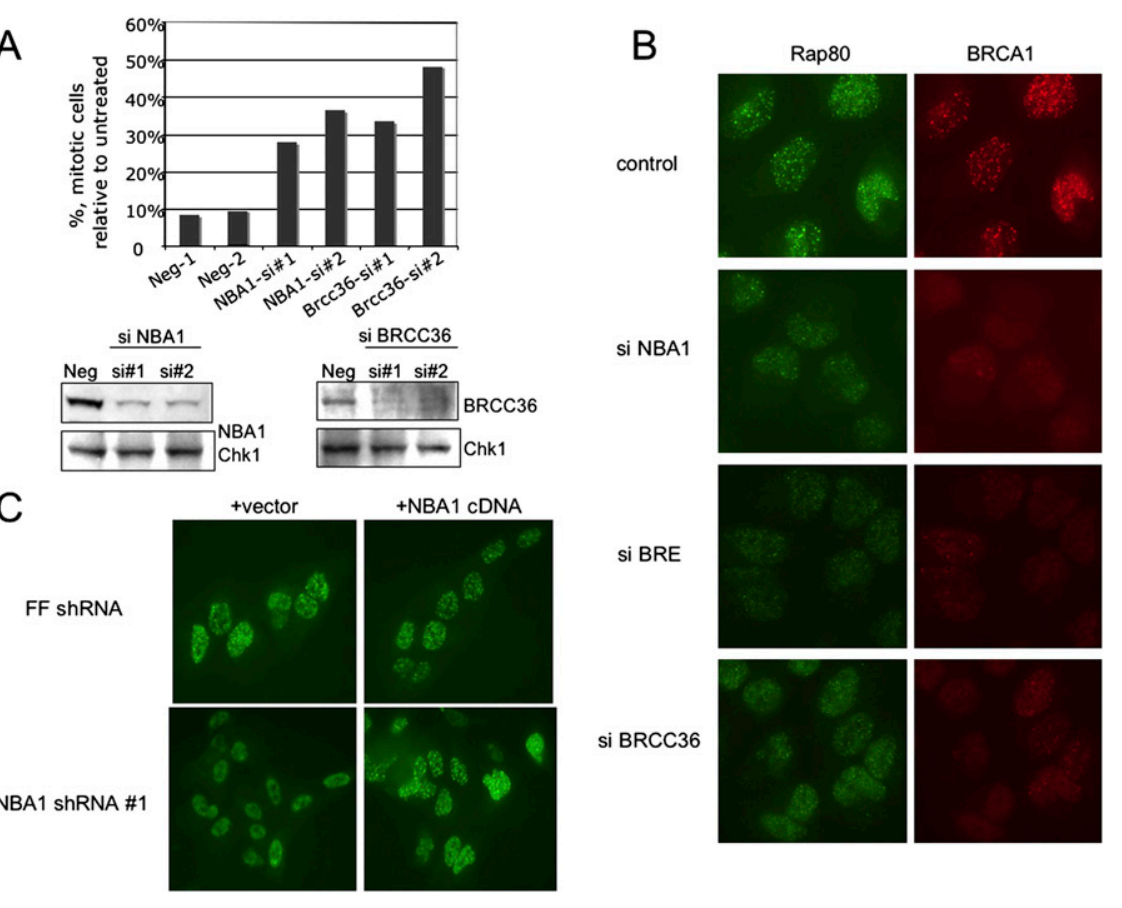

NBA1 was a member of the BRCA1 A complex and, similar to all known A complex members, is required for optimal recruitment of the BRCA1/BARD1 E3 ligase to sites of DNA damage.

Abraxas appears to serve, in part, as a central scaffold protein that assembles the various components of the BRCA1 A complex. Abral has multiple protein interaction domains. NBA1 and BRE interact with Abraxas through the N-terminal ABR region of the Abral protein, which is shared with the NBA1-interacting protein Abrol, a Abral paralog. The BRCC36 protein associates with Abraxas through a central coiled-coil domain (Wang and Elledge 2007). RAP80 association with Abraxas requires $\mathrm{N}$-terminal regions of Abraxas including ABR domain and the coiled-coil domain (Wang and Elledge 2007). The BRCA1/BARD1 ubiquitin ligase associates with Abraxas through a phospho-SPxF motif at is extreme C terminus that is recognized by the BRCT repeats of BRCA1 (Wang et al. 2007). Our data, together with others (Chen et al. 2006; Kim et al. 2007; Sobhian et al. 2007; Wang et al. 2007), suggests that each member of the BRCA1 complex is required for the recruitment of the BRCA1/BARD1 ligase to DNA damage sites and is responsible for IR resistance of the cells and proper cell cycle regulation.

Both NBA1 and BRE associate with Abraxas through the $A B R$ domain. NBA1 is required to maintian the abundance of BRE and Abral as reduction of NBA1 results in a decrease in BRE and Abral levels, most likely by controlling their stability. A direct association between NBA1 and BRE is supported by the fact that they were identified as an interacting pair in a genome scale yeast two-hybrid analysis (Rual et al. 2005). Together, these data suggest that NBA1 association with BRE or Abral directly regulates the stability of these proteins.

\section{Multiple ubiquitin-binding motifs in the} BRCA1 A complex

Bioinformatics analysis revealed multiple new motifs among BRCA1 A complex components. Abral possesses an MPN domain at its $\mathrm{N}$ terminus that lacks isopeptidase activity. Abral dimerization with BRCC36 through the coiled-coil domain on each protein positions a $\mathrm{MPN}^{+}-$ MPN domain pair in the complex. In addition, another component of the A complex, BRE, contains two UEV domains that also lack a critical residue required for enzymatic activity. Abra1, Rap80, BRE, and BRCC36 all possess polyubiquitin chain-binding activity, which is likely due to their corresponding $\mathrm{MPN}^{-}$, UIMs, UEV, and $\mathrm{MPN}^{+}$ domains. The fact that only the UIM domains in this complex have the specificity to bind K63-linked polyubiquitin chains suggests that while other ubiquitin-binding proteins may help to hold the polyubiquitin chains in an appropriate position, the UIM domains of Rap80 may act as the key receptor for K63-linked polyubiqutinated substrates.

\section{The BRCA1 A complex shares similarities with the $19 S$} complex of the proteosome

The 26S proteasome contains two major subcomplexes, the $20 \mathrm{~S}$ proteolytic core particle and the $19 \mathrm{~S}$ regulatory particle. The 19S complex contains two subcomplexes, the base complex that binds and unfolds substrates (Glickman et al. 1998; Lam et al. 2002), and the lid complex that cleaves ubiquitin from substrates and may facilitate the entry of subtrates into the catalytic proteasome core (Maytal-Kivity et al. 2002; Verma et al. 2002; Yao and Cohen 2002). The proteasome lid contains nine subunits. All except one (Sem1) contain either of two structure motifs, the MPN domain or the PCI domain. Six 

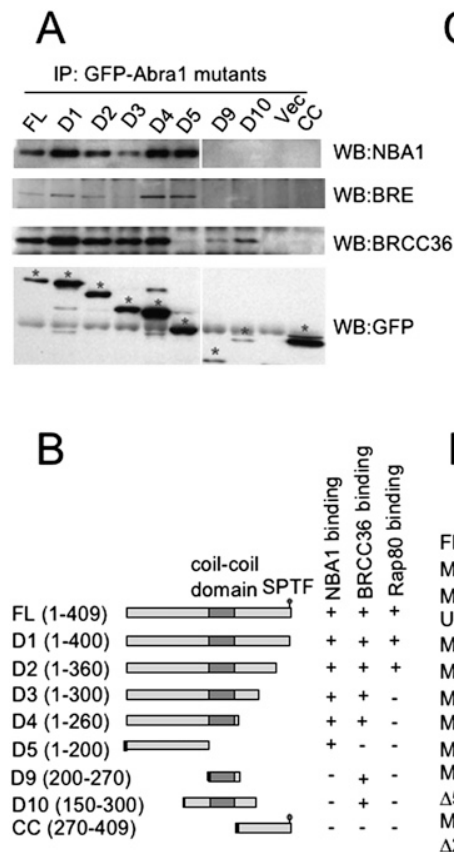

C$$
\text { 西 }
$$

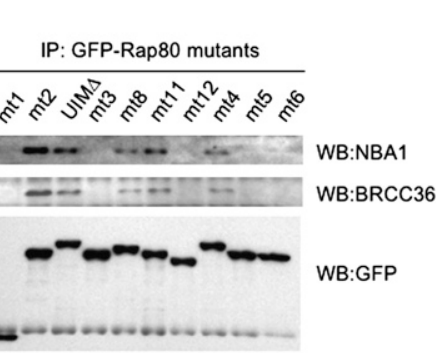

D

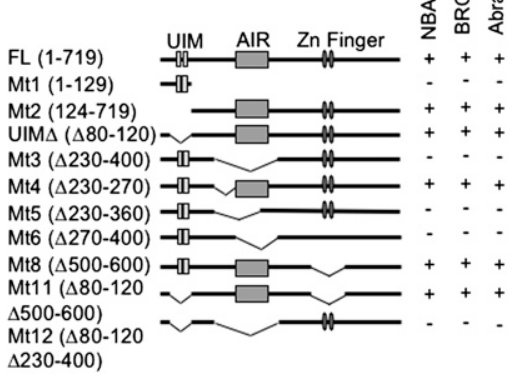

$\mathrm{E}$

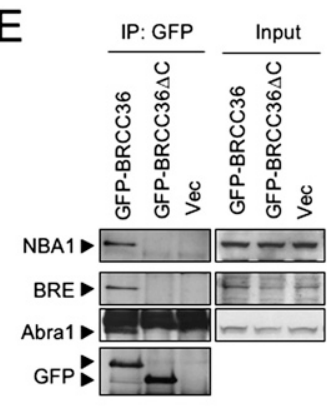

$\mathrm{F}$

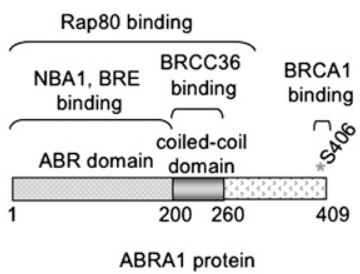

Figure 5. NBA1 serves as an adaptor protein for the BRCA1 A complex. (A) NBA1 and BRE proteins interact with the N terminus of Abra1. Cell lysates from 293T cells stably expressing GFP-tagged mutants of Abral were immunoprecipitated with GFP antibodies and immunoblot analysis was carried out with antibodies against NBA1, BRE, or BRCC36. (B) A diagram for Abral deletion mutants. (C) NBA1-Rap80 interaction, similar to BRCC36-Rap80 interaction, depends on the AIR domain of Rap80 that is required for Rap80-Abra1 interaction. Cell lysates from 293T cells stably expressing GFP-tagged mutants of Rap80 were immunoprecipitated with GFP antibodies and Western blot was carried out with antibodies against NBA1 or BRCC36. $(D)$ A diagram for Rap80 deletion mutants. $(E)$ NBA1 and BRE interaction with BRCC36 is mediated through Abral. Cell lysates from 293T cells stably expressing GFP-tagged NBA1 or BRE protein were immunoprecipitated with GFP antibodies and Western blot was carried out with antibodies against Abral, BRE, or BRCC36. $(F)$ A diagram mapping the regions of Abral that interacts with different members of the BRCA1-A complex.

subunits contain PCI domains that are thought to play a structural role allowing assembly of the lid complex, while two subunits, Rpn8 and Rpn11, contain a MPN domain. Rpn11 contains an active $\mathrm{MPN}^{+} / \mathrm{JAMM}$ motif that posess a $\mathrm{Zn}^{2+}$-dependent metalloprotease activity for substrate deubiquitination, while Rpn8 contains an enzymatically inactive $\mathrm{MPN}^{-}$domain. Two additional complexes, the Cop9 signalosome and the eIF3 complex share a similar archetecture with the proteasome.

Two components of the BRCA1 A complex contain a MPN domain, BRCC36 $\left(\mathrm{MPN}^{+}\right)$and Abral $\left(\mathrm{MPN}^{-}\right)$. This $\mathrm{MPN}^{+}-\mathrm{MPN}^{-}$domain pair (BRCC36-Abral) is also observed in both the proteasome lid complex (Rpn11-Rpn8) and the COP9 signalosome (Csn5-Csn6) (Scheel and Hofmann 2005). Unlike these, the eIF3 complex MPN-MPN pair (eIF3f-eIF3h) does not possess the isopeptidase activity. We speculate that the $\mathrm{MPN}^{+}-\mathrm{MPN}^{-}$pair may provide both affinity and help orient the ubiquitin chains to allow efficient cleavage by the $\mathrm{MPN}^{+}$metalloprotease activity.

Rap80 possesses two UIM domains at its $\mathrm{N}$ terminus, while NBA1 contains a VWA domain at its $\mathrm{N}$ terminus. Both UIM domains and a VWA domain are present in a single protein of the $19 \mathrm{~S}$ regulatory complex, Rpn10/S5a. Rpn10 contains a VWA domain at its $\mathrm{N}$ terminus and two UIM domains at its $C$ terminus and has been suggested to act as a ubiquitin receptor that, together with other ubiquitin receptor proteins, $\operatorname{Rad} 23$ and Dsk2, deliver the ubiquitinated protein to the proteasome (Elsasser and Finley 2005). The UIM domains of Rpn10 specifically bind to K48-linked polyubiquitin chains and determine the substrate specificity of the proteosome (Elsasser and Finley 2005). Similarly, the UIM domains of Rap80 provide the specificity allowing the BRCA1 A complex to bind K63-linked polyubiquitin chains.

Unlike the proteasome lid complex, the COP9 signalosome, and the eIF3 complex, the BRCA1 A complex does not contain PCI domain proteins to serve as structural organizers. Instead, Abral may play an analogous role, acting as a adaptor to organize the structure of the complex to recognize both K48- and K63-linked polyubiquitinated proteins.

What is the significance of the similarity to the 19S proteasome (Fig. 7B) and why does the BRCA1 A complex contain so many ubiquitin-binding domains? One possibility is that the different UIMs might allow integration of signals from different E3 ubiquitin ligases to control, for example, the proper localization of the BRCA1 A complex within an IRIF. Furthermore, it is unlikely that the Brcal A complex would be recruited to every K63 chain in the cell through the RAP80 UIM motifs. Perhaps these UIM domains of Rap80 do not bind K63 chains with sufficient affinity to localize the A complex to a particular K63 chain and the complex then employs additional interactions to provide specificity. The ability to interact 
A
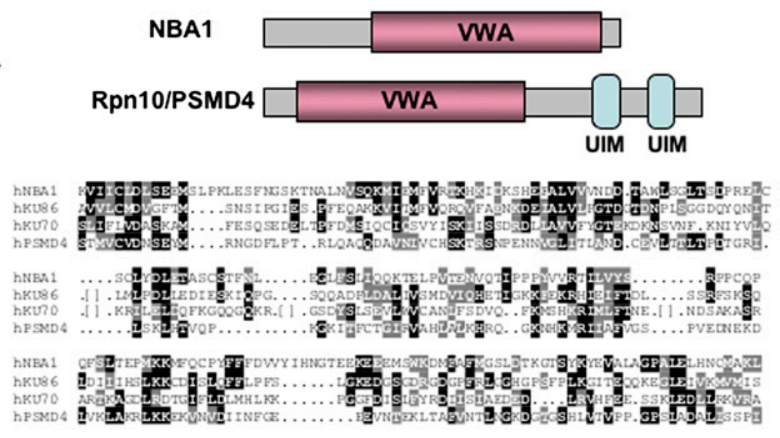

B
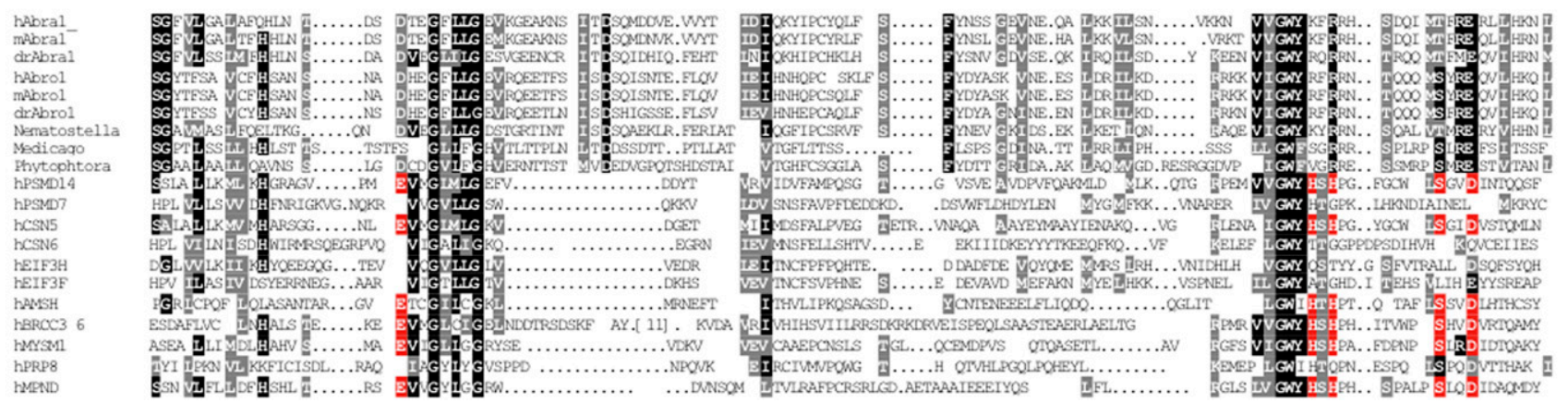

Figure 6. Members of the BRCA1 A complex contain ubiquitin-binding domains. (A) Alignment of the VWA domain of NBA1 and PSMD4/Rpn10. (B) Alignment of the MPN domains from various species of Abra1, Abro1, and other MPN domain-containing proteins. Residues outlined in red are required for enzymatic activity. (C) Alignment of the UEV domain from BRE with the UEV domain of human E2-conjugating enzyme Rad6a and UbcH5. (D) Protein domains present in the components of BRCA1 A complex. (UIM) Ubiquitin-interacting motif; (AIR) Abraxas-interacting region; (MPN-) Mpr1/Pad1 N-terminal region lacking catalytic activity; (MPN+) Mprl/Padl N-terminal region with an intact protease catalytic site; (CC) coiled coil; (UEV) ubiquitin-conjugating enzyme variant domain; (VWA) von Willebrand factor type A domain.

with multiple different $\mathrm{Ub}$ chains might provide the additional binding energy to allow the Brcal A complex to be recruited only to regions where multiple distinct $\mathrm{Ub}$ chains are located, such as sites of DNA damage. A third possibility is that once the complex is localized, these domains may be required to recognize certain ubiquitin chains in the vicinity to promote the deubiquitination of specific substrates as a further signaling event, which might be important for retaining DNA repair proteins such as BRCA1 on the damaged chromatin.

What is the role of the BRCA1 A complex in DNA repair? While the complex localizes to sites of DNA damage, its precise biochemical role there is unclear. As it has both ubiquitinating and deubiquitinating activities, it may function to amplify or remodel the existing ubiquitin chains already in place to promote DNA repair. A key to unraveling the role of this complex may reside in understanding the role of the VWA domain in NBA1. A similar VWA domain located in the DNA repair protein $\mathrm{Ku} 80$ has been speculated to act as a protein-protein interaction domain that recruits the WRN helicase to sites of damage (Doherty and Jackson 2001). Perhaps, the
NBA1 VWA domain plays a similar role to recruit repair factors to sites of damage to influence DNA repair, like the BRCA1 B and BRCA1 C complexes, which contain the helicase Brip1 and the nuclease CTIP, respectively. Alternatively, a second related VWA domain in the 19S proteosome lid appears to link the lid to the $20 \mathrm{~S}$ core particle of the proteasome responsible for proteolysis. It is possible that at some stage in the repair process, the BRCA1 A complex recruits the $26 \mathrm{~S}$ proteosome to sites of damage to promote repair or terminate structures through proteolysis. It is clear there is much to be learned about the BRCA1 A complex that is likely to shed light on the role of Brcal in DNA repair and cancer.

\section{Materials and methods}

A loss-of-function screen for radioprotective genes using shRNA libraries

The screens were performed as described previously (Schlabach et al. 2008) except cells were treated with IR or left untreated and were passaged in triplicate for $\sim 12$ population doublings (14 d) 
A

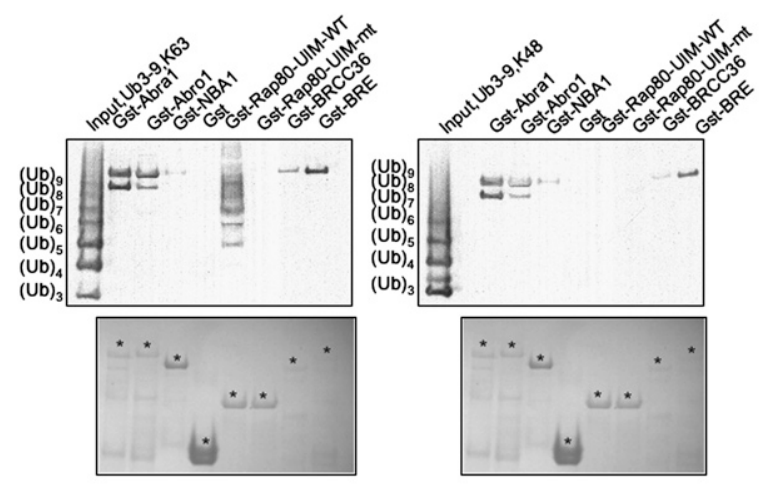

B

\begin{tabular}{llllc} 
Domain & BRCA1-A complex & 19S Proteasome & CSN & elF3 \\
\hline MPN+ & BRCC36 & Rpn11 & Csn5 & - \\
MPN & Abra1 & Rpn8 & Csn6 & elF3f,3h \\
Uev & BRE & - & - & - \\
UIM & Rap80 & Rpn10 & - & - \\
WWA & NBA1 & Rpn10 & - & - \\
PCl & - & Rpn2,3,5,6,7,9 & Csn1,2,3,4,7a,7b,8 & elF3a,3c,3e,3i,3k \\
\hline
\end{tabular}

Figure 7. BRCA1 A complex components Rap80, Abra1, BRE, and BRCC36 bind to polyubiquitin chains. (A) In vitro assay to test the ability of each member of the BRCA1-A complex to bind ubiquitin chains. The indicated GSTtagged proteins were purified from bacteria and incubated with either K63-linked polyubiquitin chains or K48-linked polyubiquitin chains. After $1 \mathrm{~h}$ incubation, the pulled-down proteins were fractionated using SDS-PAGE gel, either stained with Coomassie blue for visulization of the expression of proteins, or immunoblotted with antibodies against ubiquitin. (B) A comparison of protein domains between the BRCA1 A, 19S proteasome, CSN, and eIF3 complexes. (PCI) Proteasome/CSN/IF3 domain. and then harvested for genomic DNA isolation. Any shRNA that silences a gene necessary for repair of DNA double-strand breaks will be deleterious and will drop out of the IR-treated population and will be identified by microarray analysis (Schlabach et al. 2008). shRNAs with $\log 2$ ratios greater than 1 or less than -1 were considered enriched or depleted from the population, respectively. The details of the screens will be reported elsewhere (K. Hurov and S. Elledge, in prep.).

\section{Plasmids, siRNAs, and shRNAs}

Retroviral constructs expressing GFP-NBA1, HA-Flag-NBA1, and HA-Flag-Abral were made using a MSCV vector. Retroviral constructs expressing GFP-Rap80 and its various mutants, GFPAbral and its various mutants were described previously (Wang and Elledge 2007). Gst-Abra1, Gst-Abro1, Gst-BRCC36, GSTBRE, GST-NBA1, GST-Rap80-UIM-WT (Rap80 1-129 amino acids), and Gst-Rap80-UIM-mt (Rap80 1-129 amino acids A88S, S92A, A113S, S117A) were generated with pDEST15 vector (Invitrogen). The following siRNA used in experiments were purchased from Invitrogen: Stealth RNAi Negative Control Med GC (12935-300), Stealth RNAi for NBAl(hspc142) (HSS120850, HSS120851, HSS150852) (si1-UCAUCUUCUGG GAGACAUUGAGGGC, si2-AAUCGUCUGCACGUUCUCU GUGACC, and si3-AACAACGUCAAAGAAGAAAUAUGGG), BRCC36 (HSS128338, HSS128339, HSS128340) (si1-AAUGAA UGUGAACAUUCUGACGGC and si2-UAUGGAUUGGGAU UUCGAUUCUCUC, si3-UAAGGCUGUGGAUCCUCCUAU ACGC), BRE (HSS114298, HSS114299, HSS114300) (si1-AUUU GAAGGAUUCCAGGAGGCAAGA， si2-UUUCCAUGUUCU CUCCAUACUGUGG, and si3-AAAGCUGGGAUAUGAAGA GCUGAGG). shRNA sequences used were ATM (TTAAATGAC TGTATAGTCACCA), NBA1 sh\#1 (ATTATCCATGACAAACTAGG AG), and NBA1 sh\#2 (AAGTCCTTCCAGATTGAAGGTG)

\section{Antibodies}

NBA1 antibody was purchased from Genway (hspc142 antibody, catalog no. 15-288-22374A). Antibodies against Brcal used in this study are Brca1 D9 (Santa Cruz Biotechnologies) and Brca1 BL319 (Bethyl). Anti-GFP antibody was purchased from Invitrogen and anti-p-H3 antibody was from Upstate Biotechnologies. Other antibodies used were Rap80 antibodies (Bethyl, BL2839) BRCC36 antibodies (Invitrogen \#40-1300), BRE antibodies (Santa Cruz Biotechnologies, sc-48847; or ProteinTech Group, Inc., \#11702-1$\mathrm{AP}$ ), and ubiquitin antibodies (Santa Cruz Biotechnologies, sc9133). Polyubiquitin chains were obtained from Boston Biochem [poly-Ub(3-7)-K48-linked, UC-220; poly-Ub(3-7)-K63-linked, UC320)].

\section{Cell culture}

U2OS cells were grown in MyCoy5's medium supplemented with $10 \%$ fetal bovine serum (FBS). 293T and HeLa cells were grown in Dulbecco's modified Eagle's medium (DMEM) supplemented with $10 \%$ FBS. All cells were cultivated at $37^{\circ} \mathrm{C}$ in a humidified incubator with $5 \% \mathrm{CO}_{2}$.

\section{TAP purification}

NBA1 was cloned into a N-terminal Flag-HA-tagged doxycyclineinducible vector and transduced via retrovirus into 293T-Rex cells. Stable cells were induced with $2 \mu \mathrm{g} / \mathrm{mL}$ final concentration of doxycycline for $24 \mathrm{~h}$ followed by treatment with $10 \mathrm{~Gy}$ IR for $4 \mathrm{~h}$ before harvesting and lysates were prepped as previously described and immunoprecipitated with HA antibodies and protein was eluted with HA peptide (500 $\mu \mathrm{g} / \mathrm{mL}$ final; Sigma). The elution was TCA precipitated and analyzed by mass spectrometry. Flag-HA-tagged Abral was purified similarly, except samples were separated in a SDS-PAGE gel and gel slices were excised for mass spectrometry analysis.

\section{Cell lysis and immunoprecipitation}

Cells were lysed in NETN buffer $(50 \mathrm{mM}$ Tris- $\mathrm{HCl}$ at $\mathrm{pH}$ 8.0, 0.15 $\mathrm{M} \mathrm{NaCl}, 1 \mathrm{mM}$ EDTA, $0.5 \% \mathrm{NP}-40$ ) with protease inhibitors (Roche) and protein phosphatase inhibitors, $10 \mathrm{mM} \mathrm{NaF}$ and 50 $\mathrm{mM} \beta$-glycerophosphate. Immunoprecipitations were carried 
out in the same buffer with appropriate antibodies for $3 \mathrm{~h}$ to overnight at $4^{\circ} \mathrm{C}$.

\section{Colony-forming assay}

U2OS cells transfected with siRNAs were grown for $2 \mathrm{~d}$, then seeded at low density and irradiated with various doses of ionizing or UV radiation. Cells were left for $14 \mathrm{~d}$ at $37^{\circ} \mathrm{C}$ to allow colonies to form. Colonies were stained with $2 \%$ methylene blue $/ 50 \%$ ethanol and counted. Colonies were defined as containing 50 or more cells.

\section{G2/M checkpoint assay}

U2OS cells transfected with control oligos or siRNAs against NBA1 or BRCC36 were grown for $2 \mathrm{~d}$, irradiated with 3 Gy IR, and incubated for $1 \mathrm{~h}$. Assays were performed as described (Wang et al. 2007).

\section{Laser-induced DNA DSBS}

Cells were treated with $10 \mu \mathrm{M}$ BrdU (BD) for $24 \mathrm{~h}$ prior to laser irradiation on a Zeiss Observer.Z1 inverted microscope integrated with Palm microbeam laser microdissection workstation. Following laser treatment, the cells were incubated for $30 \mathrm{~min}$ at $37^{\circ} \mathrm{C}$, fixed, permeabilized, and stained with GFP (Invitrogen) and $\gamma$ H2AX (Upstate Biotechnologies) antibodies and Alexa 488 and Alexa 594 secondary antibodies, respectively.

\section{Color competition viability assay}

U2OS cells were retrovirally transduced with MSCV-dsRed (no selection marker) and FACs sorted to obtain a more homogeneous population. The dsRed cells were then infected with retrovirus expressing individual ATM, NBA1, or control shRNAs, selected with puromycin, and then mixed equally with U2OS cells (uncolored) expressing a control shRNA targeting FF luciferase. The mixed population was left untreated or treated with 3 Gy $y$ irradiation and following approximately six population doublings, harvested for FACs analysis. For graphical representation, the percent of dsRed-expressing cells in IRtreated samples was normalized to the behavior of untreated cells as well as to cells carrying a control shRNA (both dsRed and uncolored cells carrying the control shRNA were equally mixed). For the rescue experiment, a control FF shRNA or a NBA1 shRNA that targets the 3'UTR were introduced into dsRedexpressing U2OS cells via retrovirus and the cells were stably selected with puromycin. The cells were then infected with retrovirus that express the NBA1 cDNA and equally mixed with U2OS (uncolored) expressing a control FF shRNA and further processed as above.

\section{Immunofluoresence}

Cells were fixed with $3 \%$ paraformaldehyde/2\% sucrose for 10 min, permeablized with $0.5 \%$ Triton X-100 solution, and then immunostained with primary antibodies against various proteins and the appropriate Alexa 488-conjugated (green; Molecular Probes) and Cy3-conjugated (red; Amersham Biosciences) secondary antibodies. Images were taken with a Zeiss microscope. For the rescue experiment, a control FF shRNA or a NBA1 shRNA that targets the $3^{\prime}$ UTR were introduced into U2OS cells via retrovirus and the cells were stably selected with puromycin. The cells were subsequently infected with retrovirus expressing a NBA1 cDNA or an empty cassette and processed as above.

\section{Acknowledgments}

We thank Dr. Jianping Jin for the MSCV-N-GFP gateway expression construct, Dr. Mark Vidal for the ORFeome clones for NBA1 and BRE, C. Cotta-Ramusino for contributing to the IR screening, and M. Schlabach for help with microarray hybridizations. B.W. is a recipient of an NCI Howard Temin Award (1KO1, CA116275-01). K.H. is a Special Fellow of the Leukemia and Lymphoma Society. This work was supported by a CMCR grant (1U19A1067751-01), a DOD grant, and a NIH grant to S.J.E. S.J.E. is an investigator with the Howard Hughes Medical Institute.

\section{References}

Ambroggio, X.I., Rees, D.C., and Deshaies, R.J. 2004. JAMM: A metalloprotease-like zinc site in the proteasome and signalosome. PLoS Biol. 2: E2. doi: 10.1371/journal.pbio.0020002.

Baer, R. and Ludwig, T. 2002. The BRCA1/BARD1 heterodimer, a tumor suppressor complex with ubiquitin E3 ligase activity. Curr. Opin. Genet. Dev. 12: 86-91.

Chen, X., Arciero, C.A., Wang, C., Broccoli, D., and Godwin, A.K. 2006. BRCC36 is essential for ionizing radiation-induced BRCA1 phosphorylation and nuclear foci formation. Cancer Res. 66: 5039-5046.

Cope, G.A. and Deshaies, R.J. 2003. COP9 signalosome: A multifunctional regulator of SCF and other cullin-based ubiquitin ligases. Cell 114: 663-671.

Doherty, A.J. and Jackson, S.P. 2001. DNA repair: How Ku makes ends meet. Curr. Biol. 11: R920-R924. doi: 10.1016/ S0960-9822(01)00555-3.

Dong, Y., Hakimi, M.A., Chen, X., Kumaraswamy, E., Cooch, N.S., Godwin, A.K., and Shiekhattar, R. 2003. Regulation of BRCC, a holoenzyme complex containing BRCA1 and BRCA2, by a signalosome-like subunit and its role in DNA repair. Mol. Cell 12: 1087-1099.

Elsasser, S. and Finley, D. 2005. Delivery of ubiquitinated substrates to protein-unfolding machines. Nat. Cell Biol. 7: 742-749.

Fu, H., Reis, N., Lee, Y., Glickman, M.H., and Vierstra, R.D. 2001. Subunit interaction maps for the regulatory particle of the $26 \mathrm{~S}$ proteasome and the COP9 signalosome. EMBO J. 20: 7096-7107.

Glickman, M.H., Rubin, D.M., Coux, O., Wefes, I., Pfeifer, G., Cjeka, Z., Baumeister, W., Fried, V.A., and Finley, D. 1998. A subcomplex of the proteasome regulatory particle required for ubiquitin-conjugate degradation and related to the COP9signalosome and eIF3. Cell 94: 615-623.

Greenberg, R.A., Sobhian, B., Pathania, S., Cantor, S.B., Nakatani, Y., and Livingston, D.M. 2006. Multifactorial contributions to an acute DNA damage response by BRCA1/BARD1containing complexes. Genes \& Dev. 20: 34-46.

Hamazaki, J., Sasaki, K., Kawahara, H., Hisanaga, S., Tanaka, K., and Murata, S. 2007. Rpn10-mediated degradation of ubiquitinated proteins is essential for mouse development. Mol. Cell. Biol. 27: 6629-6638.

Harper, J.W. and Elledge, S.J. 2007. The DNA damage response: Ten years after. Mol. Cell 28: 739-745.

Hashizume, R., Fukuda, M., Maeda, I., Nishikawa, H., Oyake, D., Yabuki, Y., Ogata, H., and Ohta, T. 2001. The RING heterodimer BRCA1-BARD1 is a ubiquitin ligase inactivated by a breast cancer-derived mutation. J. Biol. Chem. 276: 14537-14540.

Hofmann, K. and Bucher, P. 1998. The PCI domain: A common theme in three multiprotein complexes. Trends Biochem. Sci. 23: 204-205. 
Hofmann, K. and Falquet, L. 2001. A ubiquitin-interacting motif conserved in components of the proteasomal and lysosomal protein degradation systems. Trends Biochem. Sci. 26: 347350.

Huen, M.S., Grant, R., Manke, I., Minn, K., Yu, X., Yaffe, M.B., and Chen, J. 2007. RNF8 transduces the DNA-damage signal via histone ubiquitylation and checkpoint protein assembly. Cell 131: 901-914.

Hurley, J.H., Lee, S., and Prag, G. 2006. Ubiquitin-binding domains. Biochem. J. 399: 361-372.

Kim, H., Chen, J., and Yu, X. 2007. Ubiquitin-binding protein RAP80 mediates BRCA1-dependent DNA damage response. Science 316: 1202-1205.

Kolas, N.K., Chapman, J.R., Nakada, S., Ylanko, J., Chahwan, R., Sweeney, F.D., Panier, S., Mendez, M., Wildenhain, J., Thomson, T.M., et al. 2007. Orchestration of the DNAdamage response by the RNF8 ubiquitin ligase. Science 318: $1637-1640$.

Lam, Y.A., Lawson, T.G., Velayutham, M., Zweier, J.L., and Pickart, C.M. 2002. A proteasomal ATPase subunit recognizes the polyubiquitin degradation signal. Nature 416: 763767.

Mailand, N., Bekker-Jensen, S., Faustrup, H., Melander, F., Bartek, J., Lukas, C., and Lukas, J. 2007. RNF8 ubiquitylates histones at DNA double-strand breaks and promotes assembly of repair proteins. Cell 131: 887-900.

Maytal-Kivity, V., Reis, N., Hofmann, K., and Glickman, M.H. 2002. $\mathrm{MPN}^{+}$, a putative catalytic motif found in a subset of MPN domain proteins from eukaryotes and prokaryotes, is critical for Rpn11 function. BMC Biochem. 3: 28. doi: 10.1186/1471-2091-3-28.

Narod, S.A. and Foulkes, W.D. 2004. BRCA1 and BRCA2: 1994 and beyond. Nat. Rev. Cancer 4: 665-676.

Paddison, P.J., Silva, J.M., Conklin, D.S., Schlabach, M., Li, M., Aruleba, S., Balija, V., O'Shaughnessy, A., Gnoj, L., Scobie, K., et al. 2004. A resource for large-scale RNA-interferencebased screens in mammals. Nature 428: 427-431.

Rual, J.F., Venkatesan, K., Hao, T., Hirozane-Kishikawa, T., Dricot, A., Li, N., Berriz, G.F., Gibbons, F.D., Dreze, M., Ayivi-Guedehoussou, N., et al. 2005. Towards a proteomescale map of the human protein-protein interaction network. Nature 437: 1173-1178.

Ruffner, H., Joazeiro, C.A., Hemmati, D., Hunter, T., and Verma, I.M. 2001. Cancer-predisposing mutations within the RING domain of BRCA1: Loss of ubiquitin protein ligase activity and protection from radiation hypersensitivity. Proc. Natl. Acad. Sci. 98: 5134-5139.

Scheel, H. and Hofmann, K. 2005. Prediction of a common structural scaffold for proteasome lid, COP9-signalosome and eIF3 complexes. BMC Bioinformatics 6: 71. doi: 10.1186/1471-21056-71.

Schlabach, M.R., Luo, J., Solimini, N.L., Hu, G., Xu, Q., Li, M.Z., Zhao, Z., Smogorzewska, A., Sowa, M.E., Ang, X.L., et al. 2008. Cancer proliferation gene discovery through functional genomics. Science 319: 620-624.

Silva, J.M., Li, M.Z., Chang, K., Ge, W., Golding, M.C., Rickles, R.J., Siolas, D., Hu, G., Paddison, P.J., Schlabach, M.R., et al. 2005. Second-generation shRNA libraries covering the mouse and human genomes. Nat. Genet. 37: 1281-1288.

Silva, J.M., Marran, K., Parker, J.S., Silva, J., Golding, M., Schlabach, M.R., Elledge, S.J., Hannon, G.J., and Chang, K. 2008. Profiling essential genes in human mammary cells by multiplex RNAi screening. Science 319: 617-620.

Smogorzewska, A., Matsuoka, S., Vinciguerra, P., McDonald, 3rd, E.R. Hurov, K.E., Luo, J., Ballif, B.A., Gygi, S.P., Hofmann, K., D'Andrea, A.D., et al. 2007. Identification of the FANCI protein, a monoubiqutinated FANCD2 paralog required for DNA repair. Cell 129: 289-301.

Sobhian, B., Shao, G., Lilli, D.R., Culhane, A.C., Moreau, L.A., Xia, B., Livingston, D.M., and Greenberg, R.A. 2007. RAP80 targets BRCA1 to specific ubiquitin structures at DNA damage sites. Science 316: 1198-1202.

Venkitaraman, A.R. 2002. Cancer susceptibility and the functions of BRCA1 and BRCA2. Cell 108: 171-182.

Verma, R., Aravind, L., Oania, R., McDonald, W.H., Yates, III, J.R. Koonin, E.V., and Deshaies, R.J. 2002. Role of Rpn11 metalloprotease in deubiquitination and degradation by the 26S proteasome. Science 298: 611-615.

Verma, R., Oania, R., Graumann, J., and Deshaies, R.J. 2004. Multiubiquitin chain receptors define a layer of substrate selectivity in the ubiquitin-proteasome system. Cell 118: 99110.

Wang, B. and Elledge, S.J. 2007. Ubc13/Rnf8 ubiquitin ligases control foci formation of the Rap80/Abraxas/Brca1/Brcc36 complex in response to DNA damage. Proc. Natl. Acad. Sci. 104: 20759-20763.

Wang, B., Matsuoka, S., Ballif, B.A., Zhang, D., Smogorzewska, A., Gygi, S.P., and Elledge, S.J. 2007. Abraxas and RAP80 form a BRCA1 protein complex required for the DNA damage response. Science 316: 1194-1198.

Wu, L.C., Wang, Z.W., Tsan, J.T., Spillman, M.A., Phung, A., Xu, X.L., Yang, M.C., Hwang, L.Y., Bowcock, A.M., and Baer, R. 1996. Identification of a RING protein that can interact in vivo with the BRCA1 gene product. Nat. Genet. 14: 430-440.

Yan, J., Kim, Y.S., Yang, X.P., Albers, M., Koegl, M., and Jetten, A.M. 2007. Ubiquitin-interaction motifs of RAP80 are critical in its regulation of estrogen receptor $\alpha$. Nucleic Acids Res. 35: 1673-1686.

Yao, T. and Cohen, R.E. 2002. A cryptic protease couples deubiquitination and degradation by the proteasome. Nature 419: 403-407.

$\mathrm{Yu}, \mathrm{X}$. and Chen, J. 2004. DNA damage-induced cell cycle checkpoint control requires CtIP, a phosphorylation-dependent binding partner of BRCAl C-terminal domains. Mol. Cell. Biol. 24: 9478-9486.

Zhou, B.B. and Elledge, S.J. 2000. The DNA damage response: Putting checkpoints in perspective. Nature 408: 433-439. 


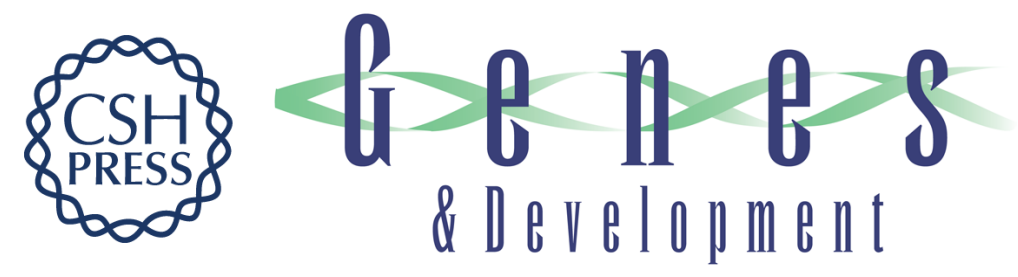

\section{NBA1, a new player in the Brca1 A complex, is required for DNA damage resistance and checkpoint control}

Bin Wang, Kristen Hurov, Kay Hofmann, et al.

Genes Dev. 2009, 23: originally published online March 4, 2009

Access the most recent version at doi:10.1101/gad.1770309

\section{Supplemental http://genesdev.cshlp.org/content/suppl/2009/03/12/gad.1770309.DC1 Material}

References This article cites 42 articles, 16 of which can be accessed free at: http://genesdev.cshlp.org/content/23/6/729.full.html\#ref-list-1

License Freely available online through the Genes \& Development Open Access option.
Email Alerting Service

